\section{SAT0361 CHEST COMPUTED TOMOGRAPHY AS AN ALTERNATIVE TO HIGH RESOLUTION MANOMETRY FOR THE DIAGNOSIS OF SCLERODERMA ESOPHAGUS}

S. Panopoulos, V.K. Bournia, G. Karamanolis, K. Denaxas, A. Zorbala, P.P. Sfikakis. First Department of Propedeutic Internal Medicine and Joint Academic Rheumatology Program, National and Kapodistrian University, Medical School, Athens, Greece

Background: Severe esophageal disease in patients with systemic sclerosis (SSc), referred as scleroderma esophagus, is characterized by ineffective or absent peristalsis along with hypotensive esophagogastric junction (hEGJ). The associations between scleroderma esophagus and different clinical and laboratory manifestations of SSc are still controversial.

Objectives: To assess associations between scleroderma esophagus, diagnosed by high resolution manometry (HRM), and other manifestations of disease.

Methods: Fifty-four consecutive SSc patients (49 women, mean age 50.6 \pm 11.6 years) with esophageal symptoms underwent clinical interview, medical records review and HRM. HRMs were analyzed according to the Chicago Classification in order to provide esophageal motility diagnosis; EGJ $<9 \mathrm{mmHg}$ was considered hypotensive. Demographic characteristics, patient-reported symptoms, SSc subtypes, pulmonary fibrosis, cutaneous ulcers, and anti-Scl-70 positivity were compared between SSc patients with or without scleroderma esophagus. Comparison was also performed in chest computed tomography (CT) findings of esophageal lumen in 26 patients with available data. Esophageal dilatation was deemed present when the diameter was $>9 \mathrm{~mm}$.

Results: Absent contractility was present in $37(68.5 \%)$ patients; among these patients hEGJP was found in 32, thus $32 / 54(59.2 \%)$ patients had classic scleroderma esophagus. There were no associations with gender, age, esophageal symptoms, skin involvement extent, anti-Scl-70, pulmonary fibrosis and cutaneous ulcers. Notably, esophageal dilation on chest CT was more frequent in patients with scleroderma esophagus compared to those without $(77 \%$ vs. $8 \%, p=0.04$, respectively).

Table 1. Associations between scleroderma esophagus and various SSc manifestations

\begin{tabular}{lccc}
\hline & $\begin{array}{c}\text { Scleroderma } \\
\text { esophagus } \\
(\mathrm{n}=32)\end{array}$ & $\begin{array}{c}\text { No scleroderma } \\
\text { esophagus } \\
(\mathrm{n}=22)\end{array}$ & $\mathrm{p}$ \\
\hline Age $(\mathrm{yrs})$ & $52.5 \pm 11.9$ & $52.5 \pm 11.9$ & \\
Sex $(\mathrm{F}, \%)$ & $28(87.5)$ & $21(98.4)$ & 0.362 \\
Diffuse SSc $(\mathrm{n}, \%)$ & $12(55.5)$ & $14(43.8)$ & 0.646 \\
Pulmonary fibrosis $(\mathrm{n}, \%)$ & $25(78.1)$ & $15(68.1)$ & 0.750 \\
Cutaneous ulcers $(\mathrm{n}, \%)$ & $19(59.4)$ & $12(54.5)$ & 0.854 \\
Anti-Scl-70 $(\mathrm{n}, \%)$ & $23(71.9)$ & $12(54.5)$ & 0.540 \\
CT coronal diameters $>9 \mathrm{~cm}(\mathrm{n}, \%)^{*}$ & $10(76.9)$ & $1(7.7 \%)$ & 0.045 \\
Presence of symptoms $(\%)$ & & & \\
- dysphagia & $18(56.3)$ & $13(39.1)$ & 0.914 \\
- heartburn & $25(78.1)$ & $21(95.4)$ & 0.620 \\
- regurgitation & $28(87.5)$ & $19(86.4)$ & 0.126 \\
- chest pain & $13(56.3)$ & $7(31.8)$ & 0.653 \\
\hline * & & &
\end{tabular}

${ }^{*}$ Data available in 26 SSc patients (13 with and 13 without scleroderma esophagus).

Conclusions: Scleroderma esophagus diagnosed by HRM was present in less than $2 / 3$ of symptomatic patients with SSc and associated only with esophageal dilation (>9mm) in CT. Although further studies are needed, esophageal dilation on chest CT may be a non-invasive alternative for evaluation of SSc patients with esophageal symptoms.

Disclosure of Interest: None declared

DOI: 10.1136/annrheumdis-2017-eular.3182

\section{SAT0362 RISK FACTORS AND OUTCOME OF THAI PATIENTS WITH SCLERODERMA RENAL CRISIS (SRC): A DISEASE DURATION-MATCHED CASE CONTROL STUDY}

S. Wangkaew $^{1}$, S. Lertthanaphok ${ }^{1}$, S. Puntana ${ }^{1}$, K. Noppakun ${ }^{2}$.

${ }^{1}$ Rheumatology; ${ }^{2}$ Nephrology, Chiang Mai University, Chiang Mai, Thailand

Background: Data regarding the prevalence, risk factors and outcome of scleroderma renal crisis (SRC) in Asian patients with systemic sclerosis (SSc) are limited.

Objectives: To determine the prevalence, risk factors and outcome of SRC in Thai SSc patients.

Methods: Medical records of all SSc patients, fulfilling the 1980 American College of Rheumatology classification criteria for SSc, seen at the Division of Rheumatology, Maharaj Nakorn Chiang Mai Hospital, Thailand, from January 1990 and December 2015 were retrospectively reviewed. Patients younger than 18 years old or overlapping with rheumatoid arthritis, systemic lupus erythematosus or mixed connective tissue disease were excluded. Cases of SRC were identified based on the international Scleroderma Renal Crisis Study criteria ${ }^{1}$. Controls were selected from consecutive SSc patients without SRC from our database that was ranked closest to the cases based on hospital number, and matched for disease duration from the first non-Raynaud's phenomenon, by \pm 1 year. The ratio for SRC patients to control patients was 1:4

Results: Of 608 SSc patients seen during the study period, 19 SRC were identified resulting of the SRC prevalence of $3.13 \%$ and there were 76 matched controls. Of the 19 cases, mean \pm SD age and median (IQR1, 3) disease duration was $56.2 \pm 13.8$ years and $9(5.0,16.0)$ months, respectively. Seventeen patients $(89.5 \%)$ had dcSSc. There were $12(63.2 \%)$ patients with hypertensive renal crisis (HRC) and 7 (16.8\%) patients with normotensive renal crisis (NRC). Univariate conditional logistic regression analyses showed that current myositis (Odd ratio [OR]21.75, 95\% Cl 2.65- 178.71, $\mathrm{p}=0.004$ ), cardiac involvement (OR5.68, 95\% Cl2.03 $-15.84, \mathrm{p}=0.001$ ), age $>60$ years (OR2.82,95\% Cl 1.01 - 7.94,p=0.049), WBC $>10,000 \mathrm{cell} / \mathrm{mm}^{3}$ (OR2.69,95\% Cl $0.99-7.26, p=0.050$ ), serum albumin $<3.0 \mathrm{mg} / \mathrm{dl}$ (OR11.08,95\% Cl $3.02-40.66, \mathrm{p}<0.001)$, and current prednisolone used $>15 \mathrm{mg} /$ day (OR19.36,95\% Cl 2.29 - 163.66, $\mathrm{p}=0.007$ ) were associated with SRC. Digital gangrene tended to show an association with SRC in the univariate analysis (OR8.00,95\% Cl0.72 - 88.22, $\mathrm{p}=0.090$ ). Variables with $\mathrm{p}<0.15$ in univariate conditional logistic regression analysis were included in the multivariate conditional logistic regression analysis. When multivariate conditional logistic regression analysis was performed, digital gangrene (adjusted odd ratio [AOR] $31.41,95 \% \mathrm{Cl} 1.16-852.23, \mathrm{p}=0.041)$, current prednisolone use $\geq 15 \mathrm{mg} /$ day (AOR31.22, 95\% Cl 1.59-613.85, $\mathrm{p}=0.024$ ), serum albumin $<3 \mathrm{mg} / \mathrm{dl}$ (AOR7.97, $95 \% \mathrm{Cl} 1.49-42.56, \mathrm{p}=0.015$ ) and cardiac involvement (AOR 6.62,95\% Cl1.08 $-40.63, p=0.041$ ) were confirmed to be independent risk factors for SRC. During a median (IQR 1, 3) follow-up of $1(0,2)$ months, $15(78.9 \%)$ patients required hemodialysis, including 9 of $12(75.0 \%)$ patients with HRC and 6 of $7(85.7 \%)$ with NRC. Twelve (63.2\%) patients received ACEI, including 9 of $12(75.0 \%)$ patients with $\mathrm{HRC}$ and 3 of $7(42.9 \%)$ patients with NRC. Ten $(52.6 \%)$ patients died, including 5 of 12 patients (41.7\%) with HRC and 5 of $7(71.4 \%)$ patients with NRC. Conclusions: SRC was an uncommon complication in Thai patients with SSc, but associated with high mortality. Digital gangrene, current prednisolone used $\geq 15$ $\mathrm{mg} /$ day, serum albumin $<3 \mathrm{mg} / \mathrm{dl}$ and cardiac involvement were independent risk factors of SRC.

References:

[1] Hudson M, et al.Semin Arthritis Rheum 2014.

Disclosure of Interest: None declared

DOI: 10.1136/annrheumdis-2017-eular.1903

\section{SAT0363 INTERSTITIAL LUNG DISEASE IN IDIOPATHIC INFLAMMATORY MYOPATHIES: A REPORT FROM THE REMICAM REGISTRY}

T. Cobo-lbáñez ${ }^{1}$, F.J. López-Longo ${ }^{2}$, B. Joven ${ }^{3}$, V. Maldonado ${ }^{4}$, I. Llorente ${ }^{5}$, C. Barbadillo ${ }^{6}$, A. Gómez Gómez ${ }^{7}$, L. Barrio Nogal ${ }^{8}$, R. Almodóvar ${ }^{9}$, L. Lojo ${ }^{10}$, L. Ruiz ${ }^{11}$, M.J. García de Yébenes ${ }^{12}$, L. Nuño ${ }^{13}$ on behalf of REMICAM. ${ }^{1} \mathrm{H}$. Infanta Sofía, Universidad Europea; ${ }^{2} \mathrm{H}$. Gregorio Marañón; ${ }^{3} \mathrm{H}$. Doce de Octubre; ${ }^{4} \mathrm{H}$. Ramón y Cajal; ${ }^{5} \mathrm{H}$. la Princesa; ${ }^{6} \mathrm{H}$. Puerta de Hierro; ${ }^{7} \mathrm{H}$. HM Sanchinarro; ${ }^{8} \mathrm{H}$. Príncipe de Asturias; ${ }^{9} \mathrm{H}$. Fundación Alcorcón; ${ }^{10} \mathrm{H}$. Infanta Leonor; ${ }^{11} \mathrm{H}$. Niño Jesús; ${ }^{12}$ Instituto de Salud Musculoesquelética; ${ }^{13} \mathrm{H}$. la Paz, Madrid, Spain

Background: Interstitial lung disease (ILD) in idiopathic inflammatory myopathies (IIM) may appear at any time in evolution and is associated with a worse prognosis. Objectives: To investigate sociodemographics data and clinical characteristics of patients with IIM and ILD from the REMICAM registry.

Methods: A multicenter retrospective study (1980-2014) was performed. Patients were classified as polymiosytis (PM), dermatomyositis (DM), IIM with antisynthetase antibodies (AB) and overlap syndrome. In addition, ILD was classified according to HRCT pattern: usual interstitial pneumonia (IP), non specific IP, organizing pneumonia, and acute IP. We compared sociodemographic data, clinical characteristics, $A B$ and treatments of patients with and without ILD, patients with ILD according to subgroup of myositis or ILD.

Results: 478 patients were included, of whom 129 (27\%) had ILD. Patients with ILD had a higher age at diagnosis, ESR and CRP and worse initial respiratory function. They were characterized by increased frequency of arthritis, systemic and cardiac manifestations, Raynaud, cardiovascular disease, pulmonary hypertension, ischemic ulcers, sclerodactyly and anti-synthetase $A B(p<0.001)$. The differences among patients with ILD were: less arthritis in PM, greater frequency of Raynaud and sclerodactyly in overlap and antisynthetase syndrome, more anti-RNP $A B$ in the overlap syndrome, more anti-synthetase and anti-Ro $A B$ in the antisynthetase syndrome, and a higher prevalence of cutaneous signs and mechanical hands in DM $(p<0.001)$. There were no differences according to the type of ILD or treatments.

Conclusions: We found some clinical manifestations and $A B$ that may help for detection of patients with IIM associated to ILD, as well as clinical manifestations to better differentiate myositis subtypes in patients with ILD.

Disclosure of Interest: None declared

DOI: 10.1136/annrheumdis-2017-eular.3268

\section{SAT0364 HISTORY OF DIGITAL ULCERS IN PATIENTS WITH SYSTEMIC SCLEROSIS IS ASSOCIATED WITH PERIPHERAL VASCULOPATHY AS ASSESSED BY VIDEOCAPILLAROSCOPY AND 22-MHZ POWER DOPPLER ULTRASONOGRAPHY}

T. Schioppo $^{1,2}$, A. Orenti ${ }^{1,3}$, P. Boracchi ${ }^{1,3}$, O. De Lucia ${ }^{2}$, A. Murgo $^{2}$, P.L. Meroni ${ }^{1,2}$, F. Ingegnoli ${ }^{1,2}$ on behalf of OPERA study group. ${ }^{1}$ Università degli Studi di Milano; ${ }^{2}$ ASST Pini-CTO; ${ }^{3}$ Lab of Medical Statistics, Epidemiology and Biometry GA Maccacaro, Milan, Italy

Background: Vasculopathy is considered a primary pathogenic event in systemic 
sclerosis (SSc) and it substantially contributes to prominent features of the disease such as digital ulcers (DUs). History of DUs (HDUs) has been shown to correlate with disease severity, new cardiovascular events, new DUs and overall poor prognosis [1]. Microvascular abnormalities as assessed by nailfold videocapillaroscopy (NVC) and power Doppler ultrasound (PDUS) have been demonstrated to be predictive of new DUs occurrence $[2,3]$.

Objectives: To study the severity of microvascular involvement at the 3rd and 4th finger of the dominant hand in patients with SSc with or without HDUs as assessed by NVC and 22-MHz PDUS.

Methods: 100 SSc consecutive patients fulfilling the 2013 EULAR classification criteria were enrolled. PDUS was performed at the 3rd and 4th finger of the dominant hand after exclusion of ulnar artery occlusion (UAO). In case of UAO non-dominant hand was examined. Ultrasound investigation was performed with Esaote MyLab 70 XVG by means of linear array transducer (10-22 MHz). Power Doppler settings were standardized (Doppler frequency $14.3 \mathrm{MHz}$, Gain $55 \%$, PRF $750 \mathrm{~Hz}$ ). PDUS measurements included sagittal scan of nailbed and fingertip qualitatively graded from 1 (no signal) to 4 (marked hyperemia) [4], and resistivity index (RI) of ulnar and radial proper digital arteries. Capillary density (number $/ \mathrm{mm}$ ) was calculated by NVC with magnification 200X performed on two images of the same digits examined by PDUS.

Results: 100 SSc patients, 87 (87\%) women, 86 (86\%) limited cutaneous SSc, median age 62.2 years old, median disease duration 8 years were evaluated. 7 $(7 \%)$ patients had UAO. $33(33 \%)$ had HDUs among them 23 had experienced more than one DUs and 2 had active DUs at the moment of evaluation. Semiquantitative perfusion score of sagittal scan of nailbed and fingertip were not significantly associated with the presence of $\mathrm{HDU}$. RI and capillary density were significantly different in the two groups as shown in the table below:

\begin{tabular}{lccc}
\hline & Presence of HDUs & Absence of HDUs & Mean difference $(95 \% \mathrm{Cl})$ \\
\hline Capillary density & 3.023 & 3.884 & $-0.862(-1.390,-0.333)$ \\
RI & 0.740 & 0.792 & $-0.052(-0.090,-0.014)$ \\
\hline
\end{tabular}

Conclusions: A significant lower RI of ulnar and radial proper digital arteries reported in patient with HDUs is novel. By contrast PDUS grading of nailfold and fingertip were not significantly different in patients with or without HDUs. The finding of a significant lower capillary number assed by NVC in patients with HDUs is consistent with previous results [2]. Adequately longitudinal studies exploring the predictive value of PDUS parameters are required to fully ascertain its role in SSc.

\section{References:}

[1] Mihai C et al. Ann Rheum Dis. 2016;75(4):681-6.

[2] Cutolo M et al. Arthritis Rheumatol. 2016;68(10):2527-39.

[3] Lescoat A et al. Arthritis Care Res. 2016;Epub ahead of print.

[4] Newman JS et al. Radiology. 1996,198:582-584.

Disclosure of Interest: None declared

DOI: 10.1136/annrheumdis-2017-eular.3844

\section{SAT0365 MUSCULOSKELETAL US AND MRI FINDINGS IN JUVENILE SCLERODERMA}

M. Tsinti, V. Dermentzoglou, E. Tsitsami. Pediatric Rheumatology Unit, 1st Department of Pediatrics, University of Athens, Children's Hospital "Aghia Sofia", Athens, Greece

Background: Musculoskeletal (MS) involvement and clinically evident arthritis occurs in up to $65 \%$ of patients with Juvenile Systemic or localized Scleroderma (JSc). It may be the first manifestation preceding even the onset of Raynaud or skin manifestations; patients presenting with arthritis, tenosynovitis or enthesistis may suffer from Sc. On the other hand clinical examination often underestimates MS involvement in Sc. US and MRI can help distinguish arthritis with effusion from dry tenosynovitis of Sc, define whether loss of range of motion (LOM) derives only from skin thickening or from bone and joint involvement and monitor disease progression.

Objectives: To describe the spectrum of MRI and US features in juvenile scleroderma with musculoskeletal involvement.

Methods: We describe MRI and color Doppler MSUS findings of clinically affected (with arthritis and/or LOM and/or overlying skin with edema or sclerosis) lower or upper extremities from 4 males and 2 females;2 with systemic sc (ssc), 2 with linear scleroderma, 1 with generalized morphea, 1 with mixed morphea (median age 8,5 years, range 7-10,5; median time from symptom onset to MRI 11 months, range 2-24). MRI sequences;T1, fluid-sensitive, and T1-FS contrast-enhanced. Comparisons were made to uninvolved areas of the extremity, and the contralateral extremity. Findings guiding diagnosis and evaluation of disease extent are depicted in the Figure.

Results: Thickening of the dermis and infiltration of the subcutaneous fat with increase in signal intensity on fluid sensitive sequences and contrastenhanced T1w images and hypointense signal lesions on unenhanced T1w images (asterisks) was apparent in 4 patients. In 2 male patients with generalized scleroderma, clinical LOM of fingers and wrists preceded skin sclerosis by 2 months. Joint and tendon sheath synovitis, indicated by initial MSUS, was detected in fluid sensitive and T1w enhanced images (white arrows). The combination of tendon-sheath synovitis and muscular fascia thickening and enhancement (black arrows), and contractures (thin white arrow) very characteristic of scleroderma, helped identify sclerodermatous musculoskeletal involvement in the absence of skin induration. Focal bone marrow edema depicted as high signal intensity in fluid sensitive sequences (curved arrow) was found in 2 cases; 1 with generalized morphea without apparent overlying skin sclerodermatous lesion, 1 with linear scleroderma with atrophic lesions in all overlying structures
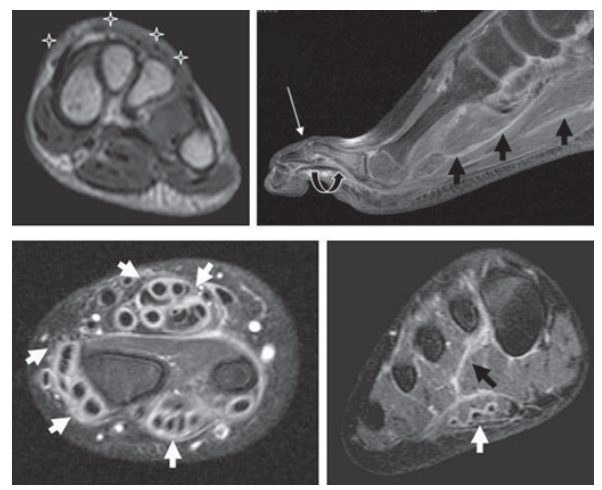

Conclusions: Musculoskeletal imaging features of juvenile scleroderma involving the skin, fascia, musculature and bones reflect pathomorphologic changes of this rare disorder and enable a complete assessment of the disease extent, including depth of infiltration and disease activity. In the described cases implementation of MSUS and MRI in Juvenile scleroderma led to earlier definition of the diagnosis and assisted the evaluation of disease extension.

References:

[1] Zulian F et al. Arthritis and Rheumatism 2007 Mar 15;57(2):203-12.

[2] Eusler E et al. Pediatr Radiol 2017 Jan 14

[3] Schannz S et al. RSNA 2011.

[4] Torok K Pediatr Clin North Am. 2012 April;59(2):381-405.

Disclosure of Interest: None declared

DOI: 10.1136/annrheumdis-2017-eular.5393

\section{SAT0366 SMOKING BEHAVIOUR AND THE PROGRESSION OF ORGAN MANIFESTATIONS IN SYSTEMIC SCLEROSIS: A LONGITUDINAL EUROPEAN SCLERODERMA TRIALS AND RESEARCH GROUP STUDY}

V.K. Jaeger ${ }^{1}$, G. Valentini ${ }^{2}$, E. Hachulla ${ }^{3}$, L. Czirják ${ }^{4}$, E. Siegert ${ }^{5}$, O. Distler ${ }^{6}$, L.M. Bambara ${ }^{7}$, E. Rosato ${ }^{8}$, Y. Allanore ${ }^{9}$, M. Matucci-Cerinic ${ }^{10}$, P. Airó ${ }^{11}$, U. Walker ${ }^{1}$ on behalf of EUSTAR co-authors. ${ }^{1}$ University Hospital Basel, Basel, Switzerland; ${ }^{2}$ Second University of Naples, Naples, Italy; ${ }^{3}$ Université de Lille, Lille, France; ${ }^{4}$ University of Pécs, Pécs, Hungary; ${ }^{5}$ University Hospital Charité, Berlin, Germany; ${ }^{6}$ University Hospital Zurich, Zurich, Switzerland; ${ }^{7}$ University of Verona, Verona; ${ }^{8}$ Sapienza University of Rome, Rome, Italy; ${ }^{9}$ Paris Descartes University, Paris, France; ${ }^{10}$ University of Florence, Florence; ${ }^{11}$ Spedali Civili, Brescia, Italy

Background: Systemic sclerosis (SSc) is a rare, multisystem autoimmune disorder. It is characterised by generalized microangiopathy, in which hypoxia and oxidative stress have been implicated in its pathogenesis. Tobacco inhalation increases free radicals and strongly promotes vascular damage. So far, data available with regards to a role of tobacco exposure with SSc severity and progression are scarce.

Objectives: We aimed to assess the effects of smoking on the speed of worsening of organ manifestations, namely lung involvement (forced vital capacity, FVC; forced expiratory volume, FEV1/FVC ratio; diffusing capacity for carbon monoxide corrected for alveolar volume, DLCO/VA), skin involvement (modified Rodnan skin score; mRSS), and digital ulcers (DU) in the European scleroderma trials and research (EUSTAR) database.

Methods: Adult SSc patients from the EUSTAR cohort with a follow-up visit $12 \pm 4$ months after their baseline visit and available data on their smoking habits were included.

The associations of smoking behaviour (never smokers vs ex-smokers vs current smokers) with the disease manifestations at follow up were assessed after adjusting for potentially confounding covariates using multivariable linear or logistic regression analyses.

Missing data were imputed using multiple imputations.

Results: Of the 3,023 patients included (mean age 57 years, SD 13; 85\% female), $66 \%$ stated that they never smoked, $23 \%$ were ex-smokers and $11 \%$ were current smokers. On average, ex-smokers had smoked for 19.5 years (SD 12) while current smokers smoked for 29.1 years (SD 13). Ex-smokers had smoked on average 17.3 pack-years (SD 20) and current smokers 29.3 pack-years (SD 36). The mean time since smoking cessation in ex-smokers was 15.8 years (SD13). The FEV1/FVC ratio changed from 96.3 (SD 14) at baseline to 96.2 (SD 13) at follow up. Taking into account the effect of age, sex, autoantibody status, disease duration and SSc subset as well as the baseline values of the outcome, the FEV1/FVC ratio decreased faster in currently smoking SSc patients than in never smokers $(\beta=-2.8, p=0.001)$. Similarly, the DLCO/VA diminished faster in current 